\title{
Tetrazole-containing derivatives of 4-amino-3-phenylbutanoic acid
}

\author{
Sergey M. Putis, Elena S. Shuvalova, and Vladimir A. Ostrovskii \\ St. Petersburg State Institute of Technology, 26, Moskowsky Av., St. Petersburg, 190013, \\ Russian Federation \\ E-mail: VA Ostrovskii@mail.ru
}

The paper is dedicated to Professor Alexander Pozharsky on his $70^{\text {th }}$ birthday

\begin{abstract}
The molecule 4-amino-3-phenylbutanoic acid contains amino and carboxy terminal groups. The reactivity of both groups was utilized for preparation of corresponding tetrazole-containing derivatives. The terminal amino group was directly replaced by a tetrazol-1-yl fragment through reaction of 4-amino-3-phenylbutanoic acid hydrochloride with triethyl orthoformate and sodium azide in acetic acid. 4-Amino-3-phenylbutanoic acid was converted into 4-(tetrazol-1-yl)-3phenylbutanoic acid and also methyl 4-(5-methyltetrazol-1-yl)-3-phenylbutanate in 79 and 45\% yields, respectively.
\end{abstract}

Keywords: Tetrazoles, 4-amino-3-phenylbutanoic acid, heterocyclization, esterification, acylation

\section{Introduction}

The tetrazole ring as an analog and metabolically stable substitute of a carboxy group is extensively used in molecular design and in the synthesis of modified amino acids and peptidomimetics. ${ }^{1}$ Certain analogs of natural amino acids containing one or several tetrazole rings have been synthesized. ${ }^{2}$

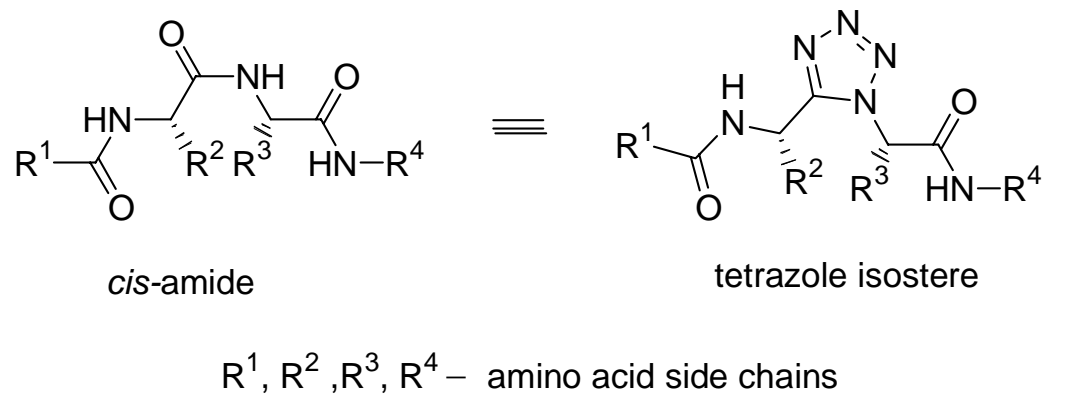


4-Aminobutanoic acid (GABA) was historically the first nootropic drug. ${ }^{3}$ 4-Amino-3phenylbutanoic 1, the corresponding hydrochloride 1a (Phenibutium), and some other derivatives of acid 1 belong to a new generation of nootropic drugs. ${ }^{4}$ The introduction of a tetrazole ring into the molecule of 4-amino-3-phenylbutanoic acid 1, and also of some derivatives of this substrate might afford promising metabolically stable analogs. We report here on the synthesis of 4(tetrazol-1-yl)-3-phenylbutanoic acid and methyl 4-(5-methyltetrazol-1-yl)-3-phenylbutanoate, the first tetrazole-containing derivatives and analogs of 4-amino-3-phenylbutanoic acid 1.

\section{Results and Discussion}

The conversion of an amino group of a primary amine into a tetrazole ring effected by a triethyl orthoformate - sodium azide system in acetic acid is well documented. ${ }^{5}$ However, this procedure was not formerly applied to the conversion of amino acids into the corresponding tetrazolecontaining derivatives. We demonstrated that the amino group of compound 1 reacted with the above-mentioned reagents to afford a tetrazole derivative, 4-(tetrazol-1-yl)-3-phenylbutanoic acid 2.

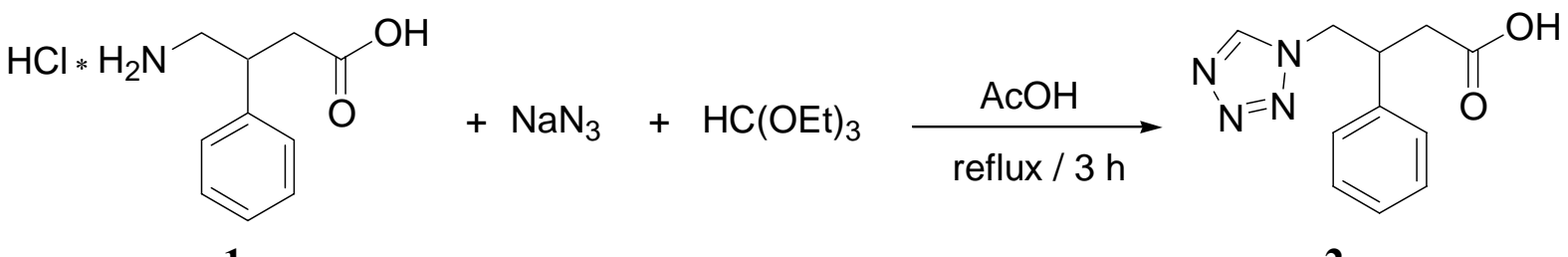

$1 \mathrm{a}$

2

\section{Scheme 1}

We also carried out an alternative way of tetrazol-1-yl substituent introduction into the structure of an ester of 4-amino-3-phenylbutanoic acid. The corresponding synthesis route was based on the conversion of primary amides into 1,5-disubstituted tetrazoles. ${ }^{2}$ In the first stage acid chloride 3 was obtained in situ and subsequently subjected to esterification into ester 4 .

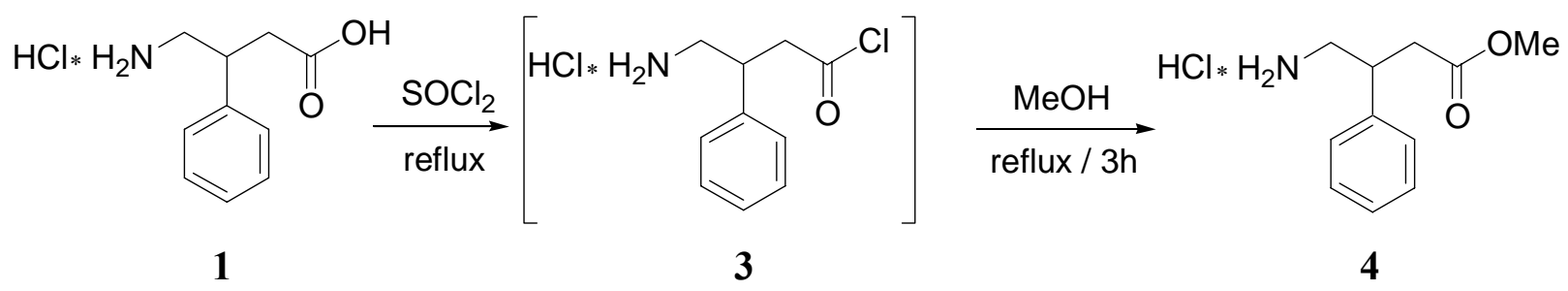

\section{Scheme 2}

In the second stage, acylation of the terminal amino group was performed in pyridine 
transforming ester $\mathbf{4}$ into amide 5.

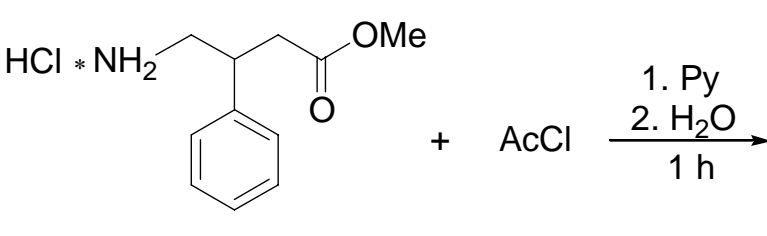

4

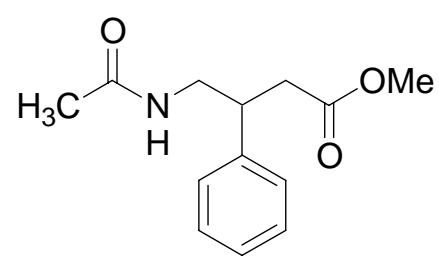

5

\section{Scheme 3}

Following the procedure, ${ }^{2}$ we succeeded in converting amide $\mathbf{5}$ into tetrazole derivative $\mathbf{6}$.<smiles>COC(=O)CC(CNC(C)=O)c1ccccc1</smiles>

5<smiles>COC(=O)CC(Cn1nnnc1C)c1ccccc1</smiles>

6

\section{Scheme 4}

Hence in this study we obtained the first representatives of tetrazole-containing analogs of 4amino-3-phenylbutanoic acid.

\section{Experimental Section}

General Procedures. ${ }^{1} \mathrm{H}$ and ${ }^{13} \mathrm{C}$ NMR spectra were recorded on a Bruker DPX-300 spectrometer. IR spectra were recorded on a SHIMADZU FTIR-8400 spectrophotometer. Elemental analysis was performed on a Hewlett-Packard $185 \mathrm{C}, \mathrm{H}, \mathrm{N}$-analyzer semi-automatic instrument. Reaction progress was monitored by TLC on Merck Kieselgel $60 \mathrm{~F}_{254}$ plates, and spots were visualized under UV light.

4-(Tetrazol-1-yl)-3-phenylbutanoic acid (2). Hydrochloride 1a (21.5 g, $0.1 \mathrm{~mol})$ and sodium azide $(7.15 \mathrm{~g}, 0.11 \mathrm{~mol})$ were added with stirring to a solution of triethyl orthoformate $(60 \mathrm{~g}, 44$ $\mathrm{ml}, 0.3 \mathrm{~mol})$ and acetic acid $(70 \mathrm{ml})$. The mixture was heated to $100{ }^{\circ} \mathrm{C}$ and kept at this temperature for $3 \mathrm{~h}$. Then the reaction mixture was cooled, filtered, and the filtrate was evaporated in a vacuum. The residue was dissolved in acetone $(100 \mathrm{ml})$, filtered, and the filtrate was evaporated in a vacuum. The residue was dissolved in distilled water $(50 \mathrm{ml})$, and a 
concentrated solution of sodium hydroxide was added thereto till $\mathrm{pH} \approx 9-10$. The solution was treated with activated carbon, filtered, and acidified with a concentrated solution of hydrochloric acid till $\mathrm{pH} \approx 2$ was reached. The precipitate was filtered off and recrystallized from ethanol to give the tetrazole $2(18.3 \mathrm{~g}, 79 \%)$, mp $175{ }^{\circ} \mathrm{C},{ }^{1} \mathrm{H}$ NMR spectrum (300 MHz, DMSO-d 6 ): $\delta$ 12.23 (brs, $1 \mathrm{H}, \mathrm{OH}), 9.01\left(\mathrm{~s}, 1 \mathrm{H}, H^{5}\right), 7.32\left(\mathrm{~m}, 5 \mathrm{H}, \mathrm{C}_{6} H_{5}\right), 4.78\left(\mathrm{~m}, 2 \mathrm{H}, \mathrm{CH}_{2}\right), 3.72$ (quintet, $J$ $8.5 \mathrm{~Hz}, 1 \mathrm{H}, \mathrm{CH}), 2.74\left(\mathrm{~m}, 2 \mathrm{H}, \mathrm{CH}_{2}\right) .{ }^{13} \mathrm{C}$ NMR spectrum $\left(75 \mathrm{MHz}, \mathrm{DMSO}-\mathrm{d}_{6}\right) \delta 172.4,151.1$, 140.0, 128.5, 127.7, 127.3, 51.9, 42.0, 37.5. IR (KBr, cm $\left.{ }^{-1}\right)$ 3126, 2985, 2929, 1708, 1456, 1260, 1139, 1072, 1018, 981, 734, 704. Anal. Calcd for $\mathrm{C}_{11} \mathrm{H}_{12} \mathrm{~N}_{4} \mathrm{O}_{2}$ (232): C, 56.89; H, 5.21; N, 24.12. Found: C, 56.75; H, 5.28; N, 24.01.

Methyl 4-amino-3-phenylbutanoate hydrochloride (4). Hydrochloride 1a (21.5 g, $0.1 \mathrm{~mol})$ was dissolved in methanol $(300 \mathrm{ml})$ at room temperature, and thionyl chloride $(17.9 \mathrm{~g}, 0.15 \mathrm{~mol})$ was added thereto at a rate maintaining a weak boiling of the reaction mixture. The reaction mixture was then heated at reflux for $3 \mathrm{~h}$ and then it was cooled to room temperature. The separated precipitate was filtered off, dried in an air flow and recrystallized from methanol to give the hydrochloride 4 (17.2 g, 75\%), Mp $159{ }^{\circ} \mathrm{C},{ }^{1} \mathrm{H}$ NMR (300 MHz, DMSO-d $\left.\mathrm{d}_{6}\right): \delta 8.21$ (brs, $\left.3 \mathrm{H}, \mathrm{NH}_{3}{ }^{+}\right), 7.30\left(\mathrm{~m}, 5 \mathrm{H}, \mathrm{C}_{6} \mathrm{H}_{5}\right), 3.80\left(\mathrm{~s}, 3 \mathrm{H}, \mathrm{CH}_{3} \mathrm{O}\right), 3.39$ (quintet, $\left.J 8.5 \mathrm{~Hz}, 1 \mathrm{H}, \mathrm{CH}\right), 2.98-2.58$ $\left(\mathrm{m}, 4 \mathrm{H}, \mathrm{CH}_{2}\right) .{ }^{13} \mathrm{C} \mathrm{NMR}\left(75 \mathrm{MHz}, \mathrm{DMSO}-\mathrm{d}_{6}\right) \delta 170.0,140.5,128.6,127.9,127.2,51.5,43.5$, 39.7, 37.9. IR (KBr, cm ${ }^{-1}$ ) 3150, 2940, 1735, 734, 704. Anal. Calcd for $\mathrm{C}_{11} \mathrm{H}_{15} \mathrm{NO}_{2} * \mathrm{HCl}(229.5)$ : C, 57.52; H, 7.02; N, 6.10. Found: C, 57.10; H, 7.23; N, 6.02.

Methyl 4-(acetylamino)-3-phenylbutanoate (5). Hydrochloride 4 (11.35 g, $0.05 \mathrm{~mol}$ ) was dissolved in dry pyridine $(50 \mathrm{ml})$ at room temperature. On cooling to $0-5{ }^{\circ} \mathrm{C}$, acetyl chloride (3.95 g, $0.05 \mathrm{~mol})$ was added dropwise and the reaction mixture was maintained at this temperature for $1 \mathrm{~h}$. Afterwards the solution was poured into an ice -water mixture $(500 \mathrm{~g})$. The precipitate was filtered off, dried in an air flow and recrystallized from ethyl ether to give the amide 5 (8 g, 68\%), Mp $41{ }^{\circ} \mathrm{C},{ }^{1} \mathrm{H}$ NMR $\left(300 \mathrm{MHz}, \mathrm{DMSO}-\mathrm{d}_{6}\right) \delta 7.91$ (brs, $\left.1 \mathrm{H}, \mathrm{NH}\right), 7.30(\mathrm{~m}$, $\left.5 \mathrm{H}, \mathrm{C}_{6} H_{5}\right), 3.46\left(\mathrm{~s}, 3 \mathrm{H}, \mathrm{CH}_{3} \mathrm{O}\right), 3.24\left(\mathrm{~m}, 3 \mathrm{H}, \mathrm{CH}+\mathrm{CH}_{2}\right), 2.68\left(\mathrm{~m}, 2 \mathrm{H}, \mathrm{CH}_{2}\right), 1.77\left(\mathrm{~s}, 3 \mathrm{H}, \mathrm{CH}_{3^{-}}\right.$ $\mathrm{C}=\mathrm{O}) .{ }^{13} \mathrm{C}$ NMR spectrum $\left(75 \mathrm{MHz}, \mathrm{DMSO}_{-} \mathrm{d}_{6}\right) \delta 172.0,169.3,142.0,128.3,127.5,126.6,51.1$, 44.0, 37.7, 22.4. IR (KBr, cm $\left.{ }^{-1}\right) 3321,3314,2998,2964,1730,1653,734,704$. Anal. Calcd for $\mathrm{C}_{13} \mathrm{H}_{17} \mathrm{NO}_{3}$ (235): C, 66.36; H, 7.28; N, 5.95. Found: C, 66.49; H, 7.23; N, 5.90.

Methyl 4-(5-methyltetrazol-1-yl)-3-phenylbutanoate (6). To a suspension of amide 5 (4.7 g, $0.02 \mathrm{~mol})$ and sodium azide $(2.6 \mathrm{~g}, 0.04 \mathrm{~mol})$ in anhydrous acetonitrile $(20 \mathrm{ml})$ was added, by small portions, a solution of $\mathrm{SiCl}_{4}(6.8 \mathrm{~g}, 0.04 \mathrm{~mol})$ in anhydrous acetonitrile $(20 \mathrm{ml})$. The reaction mixture was heated to boiling and maintained at reflux with sampling every $6 \mathrm{~h}$ to control the conversion of initial amide 5 (TLC monitoring). When initial amide 5 was found in the reaction mixture, an extra amount of the azidizing agent was added $\left(0.01 \mathrm{~mol}^{\mathrm{of}} \mathrm{NaN}_{3}\right.$ and $0.01 \mathrm{~mol}$ of $\mathrm{SiCl}_{4}$ ), and the heating was continued till complete conversion of amide 5 (TLC). On completion of the reaction the mixture was cooled to room temperature and then in small portions it was poured into a saturated solution of sodium carbonate $(250 \mathrm{ml})$ maintaining $\mathrm{pH}>7$ (CAUTION!: the formation of explosive $\mathrm{HN}_{3}$ is possible). The solution obtained was extracted with ethyl acetate $(5 \times)$. The combined organic solutions were washed with distilled water and 
dried with $\mathrm{Na}_{2} \mathrm{SO}_{4}$. Then the solvent was evaporated in a vacuum, and the residue was recrystallized from ethanol to give the tetrazole $6(2.87 \mathrm{~g}, 45 \%)$, Mp $122{ }^{\circ} \mathrm{C}$. ${ }^{1} \mathrm{H}$ NMR $(300$ MHz, DMSO-d 6 ) $\delta 7.40\left(\mathrm{~m}, 5 \mathrm{H}, \mathrm{C}_{6} H_{5}\right), 3.40\left(\mathrm{~s}, 3 \mathrm{H}, \mathrm{CH}_{3} \mathrm{O}\right), 4.73\left(\mathrm{~m}, 2 \mathrm{H}, \mathrm{CH}_{2}\right), 3.69$ (quintet, $J$ $8.5 \mathrm{~Hz}, 1 \mathrm{H}, \mathrm{CH}), 2.70\left(\mathrm{~m}, 2 \mathrm{H}, \mathrm{CH}_{2}\right), 2.40\left(\mathrm{~s}, 3 \mathrm{H}, \mathrm{CH}_{3}-\mathrm{C}^{5}\right) .{ }^{13} \mathrm{C}$ NMR $\left(75 \mathrm{MHz}, \mathrm{DMSO}-\mathrm{d}_{6}\right) \delta$ 171.5, 155.3, 142.1, 128.2, 127.6, 126.8, 52.3, 50.9, 42.1, 37.7. IR (KBr, cm $\left.{ }^{-1}\right)$ 2980, 2960, 1732, 1450, 1265, 1140, 1070, 1010, 980, 730, 700. Anal. Calcd for $\mathrm{C}_{13} \mathrm{H}_{16} \mathrm{~N}_{4} \mathrm{O}_{2}$ (260): C, 59.99; $\mathrm{H}$, 6.20; N, 21.52. Found: C, 59.20; H, 6.53; N, 21.01.

\section{References}

1. (a) Ostrovskii, V. A.; Koldobskii, G. I.; Trifonov, R. E. In Comprehensive Heterocyclic Chemistry III, Katritzky, A. R.; Ramsden, C. A.; Scriven, E. F. V.; Taylor, R. J. K., Eds.; Elsevier: Oxford, 2008; Vol. 6, p 257. (b) Herr, R. J. Bioorg. Med. Chem. 2002, 10, 3379. (c) Wittenberger S. J. Org. Prep. Proced. Int. 1994, 26, 499.

2. (a) Esikov, K. A.; Morozova, S. E.; Malin, A. A.; Ostrovskii, V. A. Russ. J. Org. Chem. (Engl. Transl.) 2002, 38, 1370. (b) Morozova, S. E.; Esikov, K. A.; Zubarev, V. Yu.; Malin, A. A.; Ostrovskii, V. A. Russ. J. Org. Chem. (Engl. Transl.) 2004, 40, 1528. (c) Morozova, S. E.; Komissarov, A. V.; Esikov, K. A.; Zubarev, V. Yu.; Malin, A. A.; Ostrovskii, V. A. Russ. J. Org. Chem. (Engl. Transl.) 2004, 40, 1532. (d) Morozova, S. E.; Esikov, K. A.; Dmitrieva, T. N.; Malin, A. A.; Ostrovskii, V. A. Russ. J. Org. Chem. (Engl. Transl.) 2004, 40, 443. (e) Ishizuka, N.; Matsumura, K.; Sakai, K.; Fujimoto, M.; Mihara, S.; Yamamori, T. J. Med. Chem. 2002, 45, 2041. (f) El-Ahl, A.-A. S.; Elmorsy, S. S.; Elbeheery, A. H.; Amer, F. A. Tetrahedron Lett. 1997, 38, 1257.

3. Kleemann, A.; Engel, J.; Kutschner, B.; Reichert, D. Pharmaceutical Substances: Syntheses, Patents, Applications, Hardback, Thieme: Stuttgart and New York, 2001; p 2488.

4. Mashkovskii, M. D. Medicines; M: NewWave, 2007; p 1206.

5. (a) Voitekhovich, S. V.; Vorobiov, A. N.; Gaponik, P. N.; Ivashkevich, O. A. Chem. Heterocycl. Compd. (Engl. Transl.) 2005, 41, 999. (b) Gaponik, P. N.; Karavai, V. P.; Davshko, I. E.; Degtyarik, M. M.; Bogatikov, A. N. Chem. Heterocycl. Compd. (Engl. Transl.) 1990, 26, 1274. 